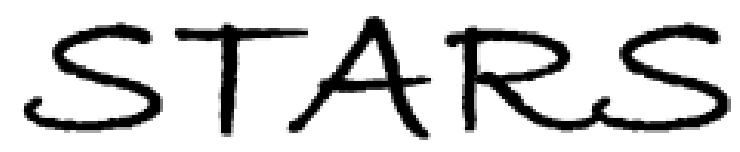

University of Central Florida

STARS

$1-1-2010$

\title{
Two-dimensional Blasius viscous flow of a power-law fluid over a semi-infinite flat plane
}

Robert A. Van Gorder

University of Central Florida

Find similar works at: https://stars.library.ucf.edu/facultybib2010 University of Central Florida Libraries http://library.ucf.edu

This Article is brought to you for free and open access by the Faculty Bibliography at STARS. It has been accepted for inclusion in Faculty Bibliography 2010 s by an authorized administrator of STARS. For more information, please contact STARS@ucf.edu.

\section{Recommended Citation}

Van Gorder, Robert A., "Two-dimensional Blasius viscous flow of a power-law fluid over a semi-infinite flat plane" (2010). Faculty Bibliography 2010s. 882.

https://stars.library.ucf.edu/facultybib2010/882

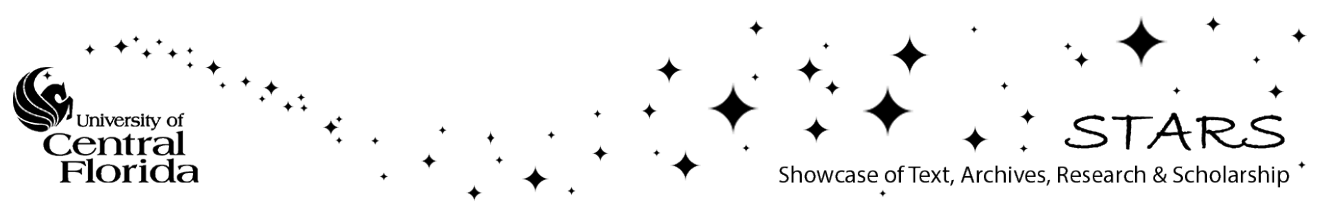




\section{Two-dimensional Blasius viscous flow of a power-law fluid over a semi-infinite flat plane}

Cite as: J. Math. Phys. 51, 112901 (2010); https://doi.org/10.1063/1.3503774

Submitted: 21 March 2010 . Accepted: 25 September 2010 . Published Online: 12 November 2010

Robert A. Van Gorder

\section{ARTICLES YOU MAY BE INTERESTED IN}

Hairpin vortex organization in wall turbulence

Physics of Fluids 19, 041301 (2007); https://doi.org/10.1063/1.2717527

Measurements of nanofluid viscosity and its implications for thermal applications

Applied Physics Letters 89, 133108 (2006); https://doi.org/10.1063/1.2356113

Flow and heat simultaneously induced by two stretchable rotating disks

Physics of Fluids 28, 043601 (2016); https://doi.org/10.1063/1.4945651

Where in the world is AIP Publishing?

Find out where we are exhibiting next 


\title{
Two-dimensional Blasius viscous flow of a power-law fluid over a semi-infinite flat plane
}

\author{
Robert A. Van Gordera) \\ Department of Mathematics, University of Central Florida, P.O. Box 161364, Orlando, \\ Florida 32816-1364, USA \\ (Received 21 March 2010; accepted 25 September 2010; published online 12 November \\ 2010)
}

\begin{abstract}
Analytic results are obtained for the similarity equation governing the twodimensional Blasius viscous flow of a power-law fluid over a semi-infinite flat plane via Taylor series for small values of the independent similarity variable. Then, an analytic perturbative procedure is used to determine an approximate solution that exhibits the correct asymptotic behavior. This perturbation method allows for the computation of the shear stress at the wall, something which is impossible with a Taylor series approach. It is found that the perturbation solutions converge sufficiently rapidly; indeed, a first order approximation gives qualitatively accurate results. Furthermore, we employ the perturbation method to deduce the influence of the power-law index, $n$, on the obtained similarity solutions. (C) 2010 American Institute of Physics.
\end{abstract} [doi:10.1063/1.3503774]

\section{INTRODUCTION}

In the present paper, the nonlinear boundary value problem governing the two-dimensional Blasius viscous flow of a power-law fluid over a semi-infinite flat plane is considered. For a thorough review of existence and uniqueness results for the solutions to the nonlinear boundary value problem, see Benlahsen et al. ${ }^{3}$ and the references therein. A related problem was also considered in Guedda and Kersner; ${ }^{7}$ in the limit $\beta \rightarrow 0$, their problem is equivalent to the power-law Blasius problem considered here.

Liao and $\mathrm{Campo}^{9}$ give an analytic approximation of temperature distributions for a laminar viscous flow over a semi-infinite plate by means of the homotopy analysis method; in their study, the flow was governed by the Blasius equation. An explicit analytic solution of the temperature distributions was obtained for general cases. Greenspan and Carrier ${ }^{6}$ considered the flow of a viscous, electrically conducting, incompressible fluid past a semi-infinite flat plate in the presence of a magnetic field which is uniform at infinity and parallel to the stream. The nonlinear governing equations were later solved analytically by Shivamoggi and Rollins. ${ }^{12}$ After deducing the asymptotic behavior of the solutions, Shivamoggi and Rollins were able to employ a perturbation method due to Bender et $a .^{2}$ in order to determine an approximate solution with the desired asymptotic behavior. Recently, Liao ${ }^{8}$ discussed multiple solutions for a related power-law Blasius problem when $n=2$. Note that in Liao, ${ }^{8}$ an absolute value was not taken in the Blasius equation, in contrast to Benlahsen et $a l .{ }^{3}$ As discussed in Benlahsen et al., ${ }^{3}$ the presence of the absolute value is relevant in order to obtain physically meaningful solutions.

We first outline the formulation of the problem governing the two-dimensional Blasius viscous flow of a power-law fluid over a semi-infinite flat plane in Sec. II. Then, in Sec. III, we provide the Taylor series solutions for a nonlinear initial value problem related to the nonlinear boundary value problem, assuming the existence of a shooting parameter, $\alpha$. By a proof given in Benlahsen et al., ${ }^{3}$ we are able to bound $\alpha$. In Sec. IV, we employ the field-theoretic perturbative procedure

\footnotetext{
a)Electronic mail: rav@knights.ucf.edu.
} 
due to Bender et al. ${ }^{2}$ to obtain analytical solutions to the nonlinear boundary value problem. From these solutions, we are able to independently obtain the values for $\alpha$. As we shall show, the values of $\alpha$ obtained via the perturbation method show good agreement with the bounds discussed in Sec. III. The field-theoretic perturbation method employed was used by Shivamoggi ${ }^{13}$ to solve the standard Blasius boundary value problem, while Shivamoggi and Rollins ${ }^{14}$ solved a coupled boundary value problem for flow and heat transfer in which the flow depends on a Blasius-like equation. In effect, we extend such results to flows governed by the power-law Blasius model. We find that the perturbation methods allow one to construct qualitatively accurate solutions in relatively few iterations.

\section{FORMULATION OF THE PROBLEM}

We consider the flow of a laminar power-law non-Newtonian fluid past a semi-infinite flat plate (the $x-y$ plane); the fluid is assumed to be incompressible. The laminar boundary-layer approximation, at constant temperature, is (Refs. 1,3,10, and 11)

$$
\begin{gathered}
\frac{\partial u}{\partial x}+\frac{\partial v}{\partial y}=0 \\
\rho\left(u \frac{\partial u}{\partial x}+v \frac{\partial u}{\partial y}\right)=\frac{\partial \tau}{\partial y},
\end{gathered}
$$

while the relevant boundary conditions are

$$
u(x, 0)=0, \quad v(x, 0)=0, \quad u(x, y) \rightarrow u_{e} \quad \text { as } \quad y \rightarrow \infty,
$$

where $u$ and $v$ are velocity components in the $x$ and $y$ directions, respectively, $\rho$ is the fluid density, and $u_{e}$ is the exterior streaming speed, which is taken to be a constant. In order to agree with the Ostwald-de Waele power-law model, the shear stress, $\tau$, is taken as

$$
\tau=\mu_{0}\left|\frac{\partial u}{\partial y}\right|^{n-1} \frac{\partial u}{\partial y},
$$

where $n>0$ is the power-law index and $\mu_{0}>0$ is the consistency. ${ }^{1,4,12,15,16}$

Let us introduce the stream function $\psi$ defined by the relations

$$
u=\frac{\partial \psi}{\partial y} \quad \text { and } \quad v=-\frac{\partial \psi}{\partial x}
$$

which allows us to reduce the boundary value problem for two functions $u$ and $v$, (1)-(3), to a boundary value problem involving only one unknown function, to wit

$$
\begin{gathered}
\frac{\partial \psi}{\partial y} \frac{\partial^{2} \psi}{\partial x \partial y}-\frac{\partial \psi}{\partial x} \frac{\partial^{2} \psi}{\partial y^{2}}=\mu_{c} \frac{\partial}{\partial y}\left(\left|\frac{\partial^{2} \psi}{\partial y^{2}}\right|^{n-1} \frac{\partial^{2} \psi}{\partial y^{2}}\right), \\
\frac{\partial \psi}{\partial y}(x, 0)=0, \quad \frac{\partial \psi}{\partial x}(x, 0)=0, \quad \frac{\partial \psi}{\partial y}(x, y) \rightarrow u_{e} \quad \text { as } \quad y \rightarrow \infty,
\end{gathered}
$$

where $\mu_{c}=\mu_{0} / \rho$. Let us consider the class of self-similar solutions of the form

$$
\psi(x, y)=\mu_{c}^{1 /(n+1)} u_{e}^{(2 n-1) /(n+1)} x^{1 /(n+1)} f(\eta), \quad \eta=\mu_{c}^{-1 /(n+1)} u_{e}^{(2 n-1) /(n+1)} x^{-1 /(n+1)} y .
$$

Under such an assumption, one obtains

$$
\begin{gathered}
\left(\left|f^{\prime \prime}\right|^{n-1} f^{\prime \prime}\right)^{\prime}+\frac{1}{n+1} f f^{\prime \prime}=0, \\
f(0)=0, \quad f^{\prime}(0)=0, \quad f^{\prime}(\infty)=1,
\end{gathered}
$$


where prime denotes differentiation with respect to $\eta$. When $n=1$, (9) reduces to the famous Blasius equation: $:^{5,11}$

$$
f^{\prime \prime \prime}+\frac{1}{2} f f^{\prime \prime}=0
$$

The analytical solution of the ordinary boundary value problem (9) and (10) shall be the focus of the present paper. Once a solution $f$ is known, the flow velocities may then be obtained from

$$
u(x, y)=u_{e} f^{\prime}(\eta), \quad v(x, y)=\frac{u_{e}^{(2 n-1) /(n+1)} \mu_{c}^{1 /(n+1)}}{n+1} x^{-n /(n+1)}\left(\eta f^{\prime}(\eta)-f(\eta)\right),
$$

while the shear stress at the wall is determined by

$$
N_{W}(x)=\mu_{0}^{1 /(n+1)} u_{e}^{(2 n+1) /(n+1)} x^{-n /(n+1)}\left|f^{\prime \prime}(0)\right|^{n-1} f^{\prime \prime}(0) .
$$

\section{POWER SERIES SOLUTION FOR A RELATED INITIAL VALUE PROBLEM}

One may assume a Taylor series solution about $\eta=0$, assuming that problems (9) and (10) are equivalent to the initial value problem

$$
\begin{gathered}
\left(\left|f^{\prime \prime}\right|^{n-1} f^{\prime \prime}\right)^{\prime}+\frac{1}{n+1} f f^{\prime \prime}=0, \\
f(0)=0, \quad f^{\prime}(0)=0, \quad f^{\prime \prime}(0)=\alpha,
\end{gathered}
$$

where $\alpha>0$ is a constant to be determined. In fact, $\alpha$ is a physical quantity, namely, the similar shear stress. ${ }^{3}$

Assuming a solution of the form

$$
f(\eta)=\frac{\alpha}{2} \eta^{2}+\sum_{k=3}^{\infty} c_{k} \eta^{k},
$$

we find that the solution to the initial value problems (14) and (15) are given by

$$
f(\eta)=\sum_{k=0}^{\infty} \frac{(-1)^{k} \alpha^{(k+1)+k(1-n)} P_{k}(n)}{n^{k}(n+1)^{k}(3 k+2) !} \eta^{3 k+2},
$$

where $P_{k}(n)$ is a polynomial in $n$. The first few values of $P_{k}(n)$ are given by

$$
P_{0}(n)=P_{1}(n)=1, \quad P_{2}(n)=21-10 n, \quad P_{3}(n)=1869-2054 n+560 n^{2} .
$$

This expression is in agreement with Blasius ${ }^{5}$ in the case of $n=1$, and for $n \geq 2$ the expression agrees with the truncated series solutions provided in Benlahsen et al. ${ }^{3}$

Assuming one can obtain a value for $\alpha$, the above expression serves as a good solution for $\eta \approx 0$. More precisely, one may compute the radius of convergence for the series solution (17), finding that the series (17) converges for $\eta$ satisfying

$$
|\eta|<\left(\sqrt[3]{3 n(n+1) \alpha^{n-2}}\right) \lim _{k \rightarrow \infty} \sqrt[3]{k^{3}\left|\frac{P_{k}(n)}{P_{k+1}(n)}\right|} .
$$

Benlahsen et $a l .{ }^{3}$ obtained bounds on the possible value of $\alpha$. In particular, they report that for all $n>0$,

$$
L(n) \stackrel{\text { def }}{=}\left(\frac{1}{6(n+1)}\right)^{\frac{1}{n+1}} \leq \alpha \leq U(n) \stackrel{\text { def }}{=}\left(\frac{1}{6 n}\right)^{\frac{1}{n+1}} .
$$

In the limit $n \rightarrow \infty$, we have that $\alpha \rightarrow 1$ from below. In Fig. 1, we show the band in which the value of $\alpha=f^{\prime \prime}(0)$ may fall. Clearly, the bound on $\alpha$ improves as $n$ increases in value. 


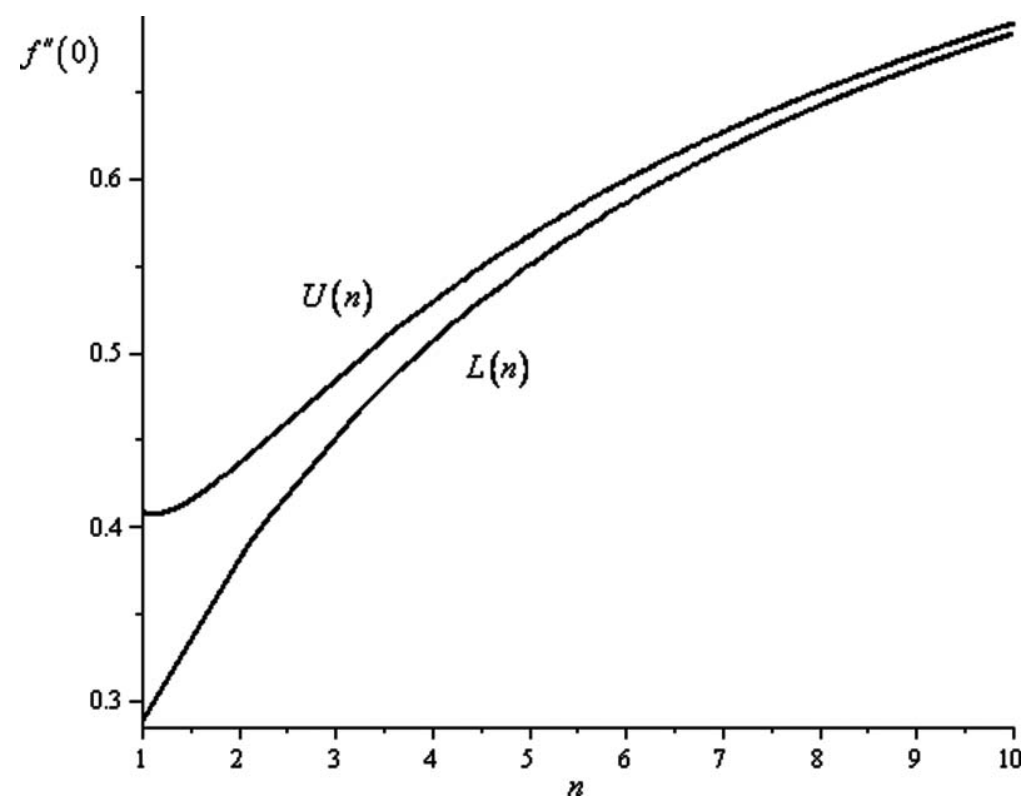

FIG. 1. Upper bound $U(n)$ and lower bound $L(n)$ on the value of the physical quantity $f^{\prime \prime}(0)$ as functions of $n$. Note that, as $n \rightarrow \infty, f^{\prime \prime}(0) \rightarrow 1$.

\section{PERTURBATION SOLUTION OVER THE SEMI-INFINITE INTERVAL}

In this section, we consider perturbation solutions to the nonlinear boundary value problems (9) and (10). In the case of $n=1$, Shivamoggi ${ }^{13}$ obtained perturbation solutions to the nonlinear boundary value problem

$$
\begin{gathered}
f^{\prime \prime \prime}(\eta)+f^{\prime \prime}(\eta) f(\eta)=0, \\
f(0)=0, \quad f^{\prime}(0)=0, \quad f^{\prime}(\infty)=1,
\end{gathered}
$$

by introducing the related problem

$$
\begin{gathered}
f^{\prime \prime \prime}(\eta)+f^{\prime \prime}(\eta)(f(\eta))^{\delta}=0, \\
f(0)=0, \quad f^{\prime}(0)=0, \quad f^{\prime}(\infty)=1,
\end{gathered}
$$

where $\delta$ is treated as a perturbation parameter. Assuming a solution of the form

$$
f(\eta)=f_{0}(\eta)+\delta f_{1}(\eta)+\delta^{2} f_{2}(\eta)+\cdots,
$$

the authors obtained the purturbation solution for (21) in the limit $\delta \rightarrow 1$. Shivamoggi and Rollins ${ }^{14}$ employed the same method in order to study the flow of a viscous, electrically conducting, incompressible fluid past a semi-infinite flat plate in the presence of a magnetic field which is uniform at infinity and parallel to the stream. Here, we employ a similar perturbation method. To this end, let us define the nonlinear boundary value problem

$$
\begin{aligned}
& \left(\left|f^{\prime \prime}\right|^{(n-1) \delta} f^{\prime \prime}\right)^{\prime}+\frac{1}{n+1} f^{\delta} f^{\prime \prime}=0, \\
& f(0)=0, \quad f^{\prime}(0)=0, \quad f^{\prime}(\infty)=1 .
\end{aligned}
$$


Clearly, when $\delta=0$, (26) becomes linear, while when $\delta=1$, we recover the original boundary value problems (9) and (10). Assuming a solution of the form (25), we find that

$$
\begin{gathered}
L\left[f_{0}(\eta)\right]=0, \\
f_{0}(0)=0, \quad f_{0}^{\prime}(0)=0, \quad f_{0}^{\prime}(\infty)=1,
\end{gathered}
$$

and

$$
\begin{gathered}
L\left[f_{j}(\eta)\right]=S_{j}(\eta), \\
f_{j}(0)=0, \quad f_{j}^{\prime}(0)=0, \quad f_{j}^{\prime}(\infty)=0
\end{gathered}
$$

for all $j=1,2,3, \ldots$, where

$$
L[y]=\frac{d^{3} y}{d \eta^{3}}+\frac{1}{n+1} \frac{d^{2} y}{d \eta^{2}}
$$

and the $S_{j}(\eta)$ 's denote the secular terms. The first two $S_{j}(\eta)$ 's are given by

$$
S_{1}(\eta)=-(n-1) f_{0}^{\prime \prime \prime}(\eta)\left\{1+\ln \left(\left|f_{0}^{\prime \prime}(\eta)\right|\right)\right\}-\frac{1}{n+1} f_{0}^{\prime \prime}(\eta) \ln \left(f_{0}(\eta)\right)
$$

and

$$
\begin{aligned}
S_{2}(\eta)= & -(n-1)\left\{\frac{f_{0}^{\prime \prime \prime}(\eta) f_{1}^{\prime \prime}(\eta)}{f_{0}^{\prime \prime}(\eta)}+f_{1}^{\prime \prime \prime}(\eta)\left\{1+\ln \left|f_{0}^{\prime \prime}(\eta)\right|\right\}\right\} \\
& -(n-1)^{2} f_{0}^{\prime \prime \prime}(\eta)\left\{\ln \left|f_{0}^{\prime \prime}(\eta)\right|+\frac{1}{2}\left(\ln \left|f_{0}^{\prime \prime}(\eta)\right|\right)^{2}\right\} \\
& -\frac{1}{n+1}\left\{f_{1}^{\prime \prime}(\eta) \ln \left(f_{0}(\eta)\right)+f_{0}^{\prime \prime}(\eta)\left\{\frac{f_{1}(\eta)}{f_{0}(\eta)}+\frac{1}{2}\left(\ln f_{0}(\eta)\right)^{2}\right\}\right\},
\end{aligned}
$$

respectively.

Now, the solution to the zeroth-order equation (28) is found to be

$$
f_{0}(\eta)=\eta-(n+1)\left\{1-\exp \left(\frac{-\eta}{n+1}\right)\right\} .
$$

Note that $f_{0}^{\prime}(\eta)=1-e^{-\eta /(n+1)}>0$ for all $\eta>0$ and $f_{0}(0)=0$, so $f_{0}(\eta)>0$ for all $\eta>0$. Hence, terms listed with $\ln f_{0}(\eta)$ exist for all $\eta>0$. Likewise, $f_{0}^{\prime \prime}(\eta)=\frac{1}{n+1} e^{-\eta /(n+1)}>0$ for all $\eta>0$, so terms listed with $\ln \left|f_{0}^{\prime \prime}(\eta)\right|$ exist for all $\eta>0$.

Inversion of the operator (32) in the linear problems (30) and (31) yields

$$
f_{j}(\eta)=\int_{0}^{\eta} \int_{0}^{\eta_{1}} \exp \left(\frac{-1}{n+1} \eta_{2}\right)\left\{\bar{S}_{j}\left(\eta_{2}\right)-\frac{\kappa_{j}}{n+1}\right\} d \eta_{2} d \eta_{1}
$$

where

$$
\bar{S}_{j}(\eta) \equiv \int_{0}^{\eta} \exp \left(\frac{1}{n+1} \tau\right) S_{j}(\tau) d \tau
$$

and

$$
\kappa_{j}=\int_{0}^{\infty} \exp \left(\frac{-1}{n+1} \tau\right) \bar{S}_{j}(\tau) d \tau .
$$

Placing the expressions (35) and (36) into (25), we see that

$$
\begin{aligned}
f(\eta)= & \eta-(n+1)\left\{1-e^{-\eta /(n+1)}\right\} \\
& +\sum_{j=1}^{\infty}\left(\int_{0}^{\eta} \int_{0}^{\eta_{1}} \exp \left(\frac{-1}{n+1} \eta_{2}\right)\left\{\bar{S}_{j}\left(\eta_{2}\right)-\frac{\kappa_{j}}{n+1}\right\} d \eta_{2} d \eta_{1}\right) \delta^{j} .
\end{aligned}
$$




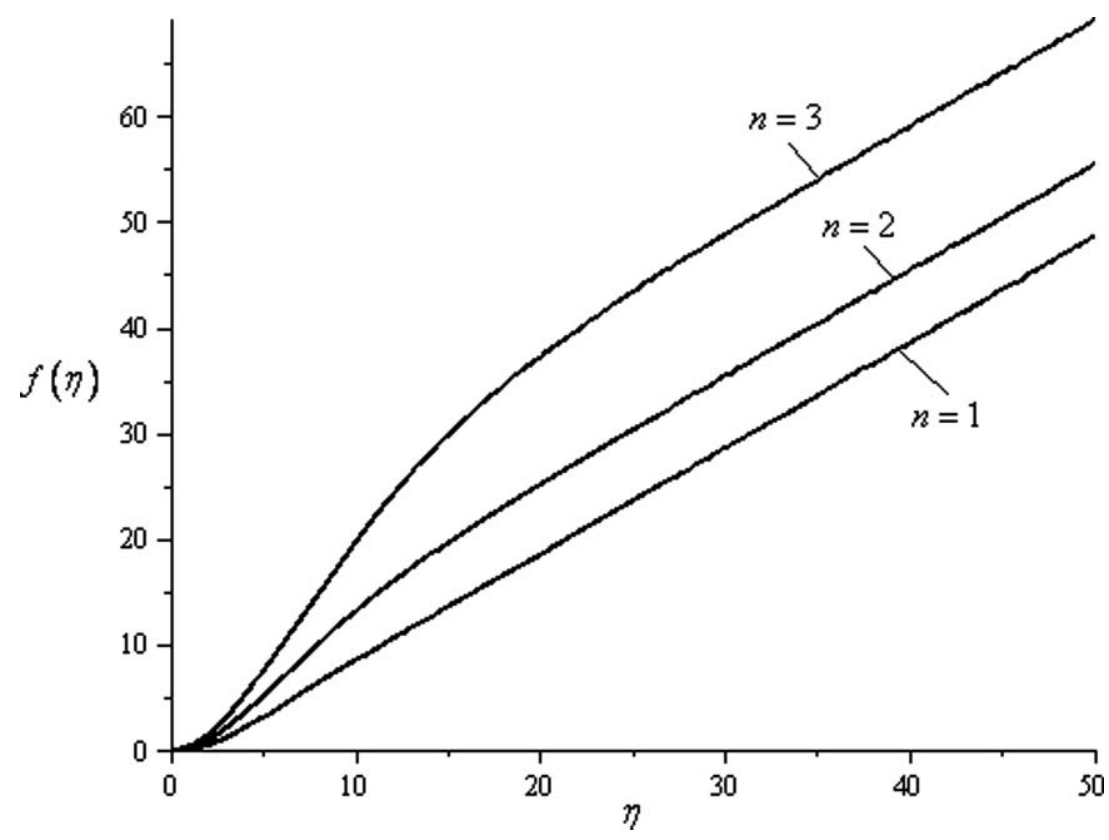

FIG. 2. Profiles of $f(\eta)$ for various values of $n$.

Differentiating (39) twice with respect to $\eta$ and then evaluating at $\eta=0$, we find that

$$
f^{\prime \prime}(0)=\frac{1}{n+1}\left\{1-\sum_{j=1}^{\infty} \kappa_{j} \delta^{j}\right\} .
$$

Returning now to the bounds on $f^{\prime \prime}(0)$ shown in Fig. 1, let us denote the quantity $\bar{\alpha}(n)$ by

$$
\bar{\alpha}(n) \stackrel{\text { def }}{=} \frac{1}{2}\{U(n)+L(n)\} .
$$

Then, $\bar{\alpha}(n)$ acts as a median possible value for the true value of $f^{\prime \prime}(0)$. As the true value of $f^{\prime \prime}(0)$ is unknown, this quantity will serve as a baseline for comparison with the perturbation results. For instance, in the case of $n=2$, we find that the value of $f^{\prime \prime}(0)$ obtained via the order $\delta$ perturbation solution [i.e., $f(\eta)=f_{0}(\eta)+f_{1}(\eta) \delta$ ] for $\delta=1$ is $f^{\prime \prime}(0) \approx 0.356$ while the relevant value of $\bar{\alpha}(n)$, for $n=2$, is $\bar{\alpha}(2)=0.409$. The relative error is then less than $13 \%$, which is not bad for only a first order approximation. Hence, we find that even the first order perturbation solution offers a reasonable approximation to $f^{\prime \prime}(0)$, though the addition of successive terms will improve the accuracy of the approximation. As one might expect, an increase in $n$ results in a decrease in the accuracy of the perturbation approximation, given a fixed number of terms, as is commonly the case when dealing with nonlinear differential equations of increasing nonlinearity. Thus, as $n$ increases in value, one needs to compute additional terms in the expansion (25) in order to attain a specific desired accuracy.

\section{RESULTS AND DISCUSSION}

In the present paper, we have provided both Taylor series and perturbation solutions for the similarity equation governing the two-dimensional Blasius viscous flow of a power-law fluid over a semi-infinite flat plane. The benefit of the Taylor series approach is that each sequential term in the expansion is easier to compute, in comparison to the perturbation method. However, the primary drawback is that the Taylor series solution (i) cannot take the boundary condition $f^{\prime}(\infty)=1$ into account and therefore (ii) one must input the value of the constant $f^{\prime \prime}(0)=\alpha>0$ in order to numerically compute a solution. In comparison, the perturbation method allows one to use the far field boundary condition $f^{\prime}(\infty)=1$ and the value of $f^{\prime \prime}(0)=\alpha$ falls out of the solution. The 


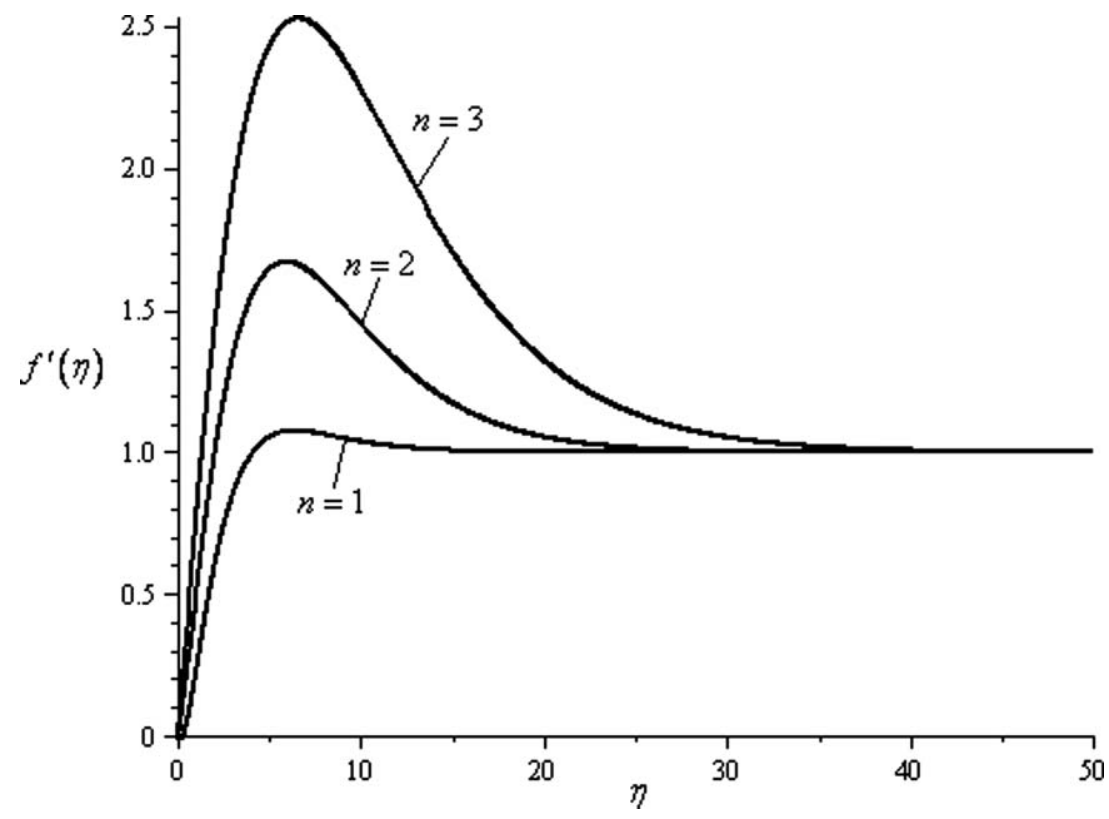

FIG. 3. Profiles of $f^{\prime}(\eta)$ for various values of $n$.

drawback is that successive terms in the approximation become quite hard to compute. In principle, one may compute the perturbation solution, obtain an approximate value of $\alpha$, and then feed this into the series solution, to obtain a good approximation valid for small $\eta$.

In Figs. 2 and 3, we plot profiles of $f(\eta)$ and $f^{\prime}(\eta)$, respectively, where the profiles are first order approximations obtained via the perturbation method. As $n$ increases in value, we see that the profiles of both $f(\eta)$ and $f^{\prime}(\eta)$ are shifted upward for all values of $\eta>0$. Interestingly, the profiles for $f^{\prime}(\eta)$ attain a maximum value $f^{\prime}\left(\eta^{*}\right)$ at some $\eta^{*}>0$ and then tend rapidly toward one as $\eta \rightarrow \infty$. Hence $f^{\prime \prime}(\eta)<0$ for all $\eta>\eta^{*}$, which again demonstrates the need for the absolute value in Eq. (9) if we want to obtain physically meaningful solutions. Such was argued in Benlahsen et al.; ${ }^{3}$ here, we arrive at the same conclusion by the use of perturbation theory.

${ }^{1}$ Acrivos, A., Shah, M. J., and Peterson, E. E., "Momentum and heat transfer in laminar boundary flow of non-Newtonian fluids past external surfaces," AIChE J. 6, 312 (1960).

${ }^{2}$ Bender, C. M., Milton, K. A., Pensky, S. S., and Simmons, L. M., Jr., "A new perturbative approach to nonlinear problems," J. Math. Phys. 30, 1447 (1989).

${ }^{3}$ Benlahsen, M., Guedda, M., and Kersner, R., "The generalized Blasius equation revisited," Math. Comput. Modell. 47, 1063 (2008).

${ }^{4}$ Bird, R. B., "Non-Newtonian behavior of polymeric liquids," Physica A 118, 3 (1988).

${ }^{5}$ Blasius, H., "Grenzschichten in Flüssigkeiten mit kleiner Reibung," Zeitschrift für Mathematik und Physik 56, 1 (1908).

${ }^{6}$ Greenspan, H. P. and Carrier, G. F., “The magnetohydrodynamic flow past a flat plate,” J. Fluid Mech. 6, 77 (1959).

${ }^{7}$ Guedda, M. and Kersner, R., "Asymptotic behavior of the unbounded solutions to some degenerate boundary layer equations revisited," Arch. Math. 89, 278 (2007).

${ }^{8}$ Liao, S., "A challenging nonlinear problem for numerical techniques," J. Comput. Appl. Math. 181, 467 (2005).

${ }^{9}$ Liao, S. and Campo, A., "Analytic solutions of the temperature distribution in Blasius viscous flow problems," J. Fluid Mech. 453, 411 (2002).

${ }^{10}$ Nachman, A. and Callegari, A., "A nonlinear singular boundary value problem in the theory of pseudoplastic fluids," SIAM J. Appl. Math. 38, 275 (1980).

${ }^{11}$ Schlichting, H. and Gersten, K., Boundary Layer Theory, 8th revised and enlarged ed., (Springer-Verlag, Berlin, 2000).

${ }^{12}$ Schowalter, W. R., "The application of boundary layer theory to power-law pseudoplastic fluids: Similar solutions," AIChE J. 6, 24 (1960).

${ }^{13}$ Shivamoggi, B. K., Perturbation Methods for Differential Equations (Birkhauser Boston, Cambridge, MA, 2003).

${ }^{14}$ Shivamoggi, B. K. and Rollins, D. K., "Magnetohydrodynamic boundary layer on a flat plate: Further analytic results," J. Math. Phys. 40, 3372 (1999).

${ }^{15}$ Tanner, R. I., Engineering Rheology (Calderon, Oxford, 1988).

${ }^{16}$ Teipel, I., "Discontinuities in boundary-layer problems of power-law fluids," Mech. Res. Commun. 1, 269 (1974). 